\title{
Unusual fatal multiple-organ dysfunction and pancreatitis induced by a single wasp sting
}

Azad C (1), Parmar VR (1), Jat KR (1)

(1) Department of Pediatrics, Government Medical College and Hospital, Chandigarh, India.

\begin{abstract}
Acute onset of multiple organ dysfunction syndrome (MODS) is a well-known complication following multiple wasp stings. However, MODS after a single wasp sting has been rarely reported in children and acute pancreatitis have probably never been observed before. Herein we describe the case of a 12-year-old boy who had urticaria and abdominal pain after a single wasp sting. The child gradually developed MODS while his abdominal complaints were worsening. Despite aggressive supportive management, the child did not survive. Afterward, the cause of the acute abdomen was finally diagnosed as acute pancreatitis. Both MODS and pancreatitis following a single wasp sting are very unusual. Thus, although pancreatitis is rarely manifested, it should be suspected after a wasp sting if there are predominant abdominal symptoms.
\end{abstract}

Key words: wasp sting, multiple organ dysfunction syndrome, pancreatitis.

\section{INTRODUCTION}

A wasp sting is a commonplace occurrence in tropical areas and sometimes may lead to serious complications including anaphylaxis, renal failure, multiple organ dysfunction syndrome (MODS) and various neurological manifestations (1-7). MODS usually occurs after multiple wasp stings and is rare after a single sting $(1,2)$. Similarly, pancreatitis is very rare after wasp sting; only one case report on an adult patient could be found (5). In the current study, we share our experience of managing a case of unusual MODS and pancreatitis in a child after a single wasp sting. To the best of our knowledge, this is the first report in which both MODS and pancreatitis occurred in the same child after a single wasp sting.

\section{CASE REPORT}

A 12-year-old boy from a rural hilly area (Kangra district of Himachal Pradesh, India; location: $30^{\circ} 22^{\prime} 40^{\prime \prime}$ to $33^{\circ} 12^{\prime} 40^{\prime \prime}$ north latitude and $75045^{\prime} 55^{\prime \prime}$ to $79^{\circ} 04^{\prime} 20^{\prime \prime}$ east longitude) was presented at the pediatric emergency unit of our hospital with history of a yellow wasp sting received 13 days earlier for which he had received no treatment. It was the month of September (post rainy season). Within hours of the wasp sting he developed fever and urticaria. The urticaria subsided in two days but the fever continued. Two days later, the child had diffuse abdominal pain and vomiting. He received local treatment for the abdominal pain but it persisted and on day seven of illness, he had melena followed by nasal bleeding after two days. Forty-eight hours before 
admission he developed excessive irritability. There was no history of jaundice, cola-colored urine, decreased urinary output or abdominal distension. Past history and family history were noncontributory.

At admission, physical examination revealed temperature of $40^{\circ} \mathrm{C}$, tachypnea and decompensated shock. There was no pallor, icterus, cyanosis, clubbing, edema, lymphadenopathy or petechiae. Respiratory and cardiovascular examinations were unremarkable. The abdomen was tender although no distension was noted. The central nervous system was normal except for marked irritability.

Complete blood counts showed $\mathrm{Hb}$ of $80 \mathrm{~g} / \mathrm{L}$, TLC of $9 \times 10^{9} / \mathrm{L}$ with $91 \%$ neutrophils, and platelet count of $140 \times 10^{9} / \mathrm{L}$. The prothrombin time index was $46 \%$. Biochemistry revealed sodium of $131 \mathrm{mmol} / \mathrm{L}$, potassium $2.4 \mathrm{mmol} / \mathrm{L}$, urea nitrogen $13.2 \mathrm{mmol} / \mathrm{L}$, creatinine $114.9 \mu \mathrm{mol} / \mathrm{L}$, aspartate aminotransferase (AST) $312 \mathrm{U} / \mathrm{L}$ and alanine aminotransferase (ALT) $118 \mathrm{U} / \mathrm{L}$. Urine examination was normal. There was no evidence of hemolysis or rhabdomyolysis. Widal test, dengue serology, viral markers of hepatitis $A$ and $\mathrm{E}$ and HIV were negative. Abdominal ultrasound showed no abnormality except for free fluid in the abdomen. Chest X-ray showed little infiltrate. Blood and urine culture were sterile.

Child managed for shock with fluid boluses and inotropes along with supportive measures. Fresh frozen plasma was infused for coagulopathy. $\mathrm{He}$ had worsening respiratory status on day two onwards that required ventilation. Abdominal pain continued after admission with further worsening in the form of abdominal distension and increase in tenderness. Then, a surgical opinion was sought. The patient underwent emergency surgery for suspected acute pathology (bowel perforation or peritonitis). Intraoperative findings showed $2 \mathrm{~L}$ of ascitic fluid which was transudative in nature; viscera were normal. Reports of serum amylase [476 U/L (normal 30$100 \mathrm{U} / \mathrm{L}$ )] and serum lipase [468 U/L (normal 145-216 U/L)], sent earlier before surgery, were received and suggested pancreatitis.

The child continued to worsen and developed acute respiratory distress syndrome; shock worsened; sensorium deteriorated; renal and liver function tests worsened and thrombocytopenia was manifested. Despite aggressive supportive management, he did not improve and died on day four of the hospital stay. Permission to perform an autopsy was refused by his parents.

\section{DISCUSSION}

The insect order Hymenoptera consists of Vespidae (hornets, wasps and yellow jackets), Apidae (honey bees and bumble bees) and Formicidae (ants). The toxic principles in hymenoptera venom include histamine, serotonin, kinins, phospholipase $\mathrm{A}_{2}\left(\mathrm{PLA}_{2}\right)$, hyaluronidase, mellitin and apamine. PLA 2 is believed to trigger the release of arachidonic acid from lipid in the cell membrane which initiates production of inflammatory eicosanoids (4). Wasp stings have been associated with a wide variety of reactions from mild local reaction to fatal systemic reactions. Generalized reactions are usually due to IgE-mediated type 1 anaphylaxis, and can occur even after a single sting. Besides the usual immediate type I hypersensitivity reactions, type III reaction with deposition of immune complexes and activation of the complement system may also contribute to the pathogenesis of the disease $(1,3)$. The management of massive wasp envenomation remains supportive with no specific antivenom being available (8).

MODS has been reported after multiple wasp stings, usually early in the course (2-4). Sharmila et al. (1) reported MODS and death after a single wasp sting in a 12-year-old boy. Our patient also had MODS after a single wasp sting and could not be saved. But the present case had delayed onset and prolonged course of MODS. Ghosh et al. (3) have reported a case of a seven-year-old girl presenting with interstitial nephritis five days after a wasp sting, and have postulated it to be caused by a type III hypersensitivity reaction.

Sharma et al. (5), from the same city, reported pancreatitis in a 60-year-old man after multiple wasp stings. Pancreatitis in the present case occurred after a single wasp sting. PLA $\mathrm{PL}_{2}$ is believed to play an important role in causing acute pancreatitis. Melittin in conjunction with $\mathrm{PLA}_{2}$ can cause membrane disruption and can act on pancreatic acinar cells to induce acute pancreatitis (5). Acute pancreatitis in our case mimicked acute abdomen, prompting surgeons to perform laparotomy.

In summary, the outcome of both MODS and pancreatitis after a single wasp sting is very unusual. Life-threatening reactions to wasp sting 
can develop even after a single sting and it may occur slowly over a time period. Pancreatitis is rare and should be suspected after a wasp sting if there are predominant abdominal symptoms.

\section{COPYRIGHT}

(C) CEVAP 2011

\section{SUBMISSION STATUS}

Received: October 10, 2010.

Accepted: December 15, 2010.

Abstract published online: January 25, 2011.

Full paper published online: February 28, 2011.

\section{CONFLICTS OF INTEREST}

There is no conflict.

\section{CORRESPONDENCE TO}

CHANDRIKA AZAD, Department of Pediatrics, Government Medical College and Hospital, Sector 32, Chandigarh, 160030, India. Phone: 0091964 6121597. Fax: 0091172 2608488. Email: chandrika_azad@yahoo.co.in.

\section{REFERENCES}

1. Sharmila RR, Chetan G, Narayanan P, Srinivasan S. Multiple organ dysfunction syndrome following single wasp sting. Indian J Pediatr. 2007;74(12):1111-2.
2. Pramanik S, Banerjee S. Wasp stings with multisystem dysfunction. Indian Pediatr. 2007;44(10):788-90.

3. Ghosh JB, Roy M, Bala AK. Delayed onset interstitial nephritis following multiple wasp stings. Indian J Nephrol. 2009;19(2):71-3.

4. Bhatta N, Singh R, Sharma S, Sinnha A, Raja S. Acute renal failure following multiple wasp stings. Pediatr Nephrol. 2005;20(12):1809-10.

5. Sharma N, Balamurugeshan PK, Sharma A. Acute pancreatitis and acute renal failure following multiple hornet stings. J Venom Anim Toxins incl Trop Dis. 2006;12(2):310-4.

6. Betten DP, Richardson WH, Tong TC, Clark RF. Massive honey bee envenomation-induced rhabdomyolysis in an adolescent. Pediatrics. 2006;117(1):231-5.

7. Hira HS, Mittal A, Kumar SA, Singh A, Ahalawat RS. Myasthenia gravis and acute respiratory muscle paralysis following wasp sting. Indian J Chest Dis Allied Sci. 2005;47(3):197-8.

8. Ferreira Junior RS, Anderlini RP, Sant'Anna OABE, Pimenta DP, Orsi RO, Barraviera B. New nanostructured silica adjuvant (SBA15) employed to produce antivenom in young sheep using Crotalus durissus terrificus and Apis mellifera venoms detoxified by cobalt-60. J Toxicol Environ Health A. 2010;73(12-14):92633. 